\title{
Speed and Position Control of Train Sytem Using GPS
}

\author{
Arshiya ${ }^{1}$, Kehkeshan Jalall $\mathrm{S}^{2}$, Sufia banu ${ }^{3}$ \\ ${ }^{l}$ (Department Electronics \& Communication, HKBK College of Engineering, India) \\ ${ }^{2}$ (Department Electronics \& Communication, HKBK College of Engineering, India) \\ ${ }^{3}$ (Department Electronics \& Communication, HKBK College of Engineering, India)
}

\begin{abstract}
The passengers face some problems regarding the arrival time of the train and they go on asking the ticket checkers about the arrival. This creates trouble to the ticket checker to keep answering thousands of passengers who are boarding the train. The GPS (Global Positioning System) gives the position of any object on which it as placed with respect to the latitude and longitude of the earth. This technique would be helpful in tracking the exact location of the train and as well the speed of the moving object. Some of the human activities causing accidents include railway level crossing during the approach of train, misplaced fish plates and high speed at places where speed should actually be less. So the system designed here which is called as ATCS (Advanced train control system) helps in reducing the accidents to an extent.
\end{abstract}

Keywords - NMEA format, GPS, RBC track switch, Tarang (Zigbee transceiver), Microcontroller, Level crossing.

\section{INTRODUCTION}

In most of the countries' railway systems, there are many accidents occurring due to human activities and natural calamities. In hilly regions, the railways are built by breaking the hills. In such places, huge boulders fall naturally on the track and hence block the way suddenly. Due to such sudden actions, there might be some chances of accidents. Also the signals at track are not seen due to foggy weather conditions due to which train accidents occur as the driver will not be able to see the signals clearly. Not only due to natural activities, but due to human activities, there are many accidents occurring too. Some of the human activities causing accidents include railway level crossing during the approach of train, misplaced fish plates and high speed at places where speed should actually be less. So the system designed here which is called as ATCS (Advanced train control system) helps in reducing the accidents to an extent.

The passengers also face some problems regarding the arrival time of the train [1] and they go on asking the ticket checkers about the arrival. This creates trouble to the ticket checker to keep answering thousands of passengers who are boarding the train. The GPS (Global Positioning System) gives the position of any object on which it as placed with respect to the latitude and longitude of the earth. This technique would be helpful in tracking the exact location of the train and as well the speed of the moving object. So this method can be used in the railway system also to get the exact position of the train and it can be displayed in the train as well as the railway station which is called as RBC section in this module.

The train monitoring system using GPS addresses issues like detecting the real time position and current speed of the train. The current system only gives the position of the train in between the two stations but can't detect the real time position of the train where it is right now. Our system receives the train's position and velocity information from the GPS. The same information is sent to the RBC[2] (Control Section) via $\mathrm{ZigBee}$ (Tarang) and speed of the train is made to increase or decrease based on algorithm provided.

What was needed in the current Indian Railways was a system that would detect the current position and the speed of the train and command the driver what speed should be maintained till the next instruction comes. The current command system uses the radio signals, which had limitations. There will be some signal distortions and irrelevant messages are heard using the walkie-talkie system which is used in the current railways systems. The TMS was a major improvement for the simple fact that it would utilize a railway map database via Global Positioning Systems to provide the driver with the exact data.

\section{MODULE FEATURES}

The system is divided into the following module and is developed in the given order:

- NMEADL: the GPS sends the data in NMEA format. The microcontroller will be programmed with decoder logic which extracts the information and converts it into a format readable by the speed calculation algorithm.

- Speed Calculating Algorithm: the RBC calculates the position and speed of the train using dead reckoning algorithm. 
- The module has an algorithm to calculate the distance at which brakes must be applied when there is a normal condition, emergency conditions and unavoidable conditions.

- Database: The position and speed of the train are found using the GPS. These coordinates are stored in the database.

- Obstacle Detection: This module contains all the necessary hardware interfaces.

- Collision avoidance: This module contains all the necessary hardware interfaces.

- Level crossing: it has a stepper motor which makes the gate closure automatic and an APR to alert the automobiles regarding the arrival of the train.

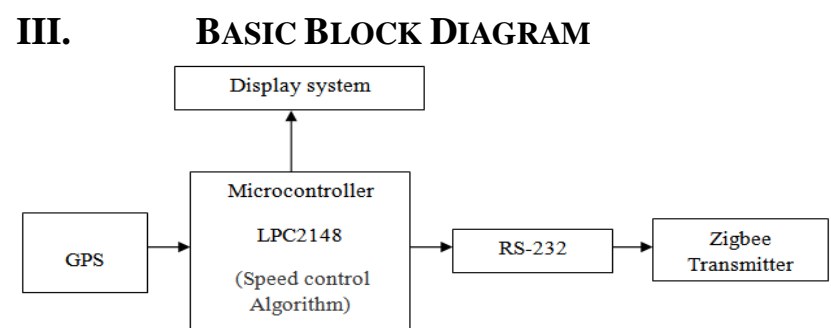

Fig.1 Train Section

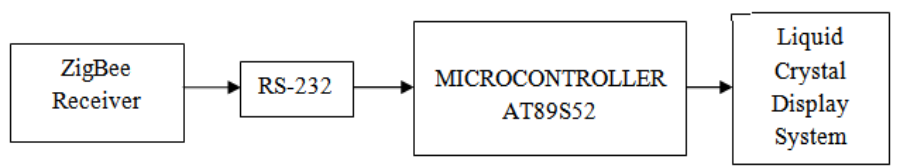

Fig.2 RBC Section

The data inputs to the controller from the GPS (Latitude, Longitude and Speed) are the major inputs and the display alerts are the outputs. This alert gives the exact location and speed at which the train is moving to the train-driver which is displayed on the on-train LCD display and will also be sent to the RBC section. All the components of the system are integrated on a single board. The hardware platform used in the system is LPC2418 and the Embedded C will be used as the working platform to program the microcontroller. The data from the GPS will be given as input to the LPC2148 which will be programmed to extract the required NMEA data format and will be converted into a form which directly displays the Latitude, Longitude, Speed and other information. This information about the location and speed of the train will also be sent to RBC section by using Zigbee which is used for the wireless transmission of the information from the train to RBC. The maximum distance between the transmitter and the receiver can go up to 300feet.At RBC the Zigbee receiver is used to receive this information which will be given to an 8051 microcontroller serially by making use RS232. This information will also be displayed on the LCD which is present at the RBC.

The GPS is used here to calculate the position and the speed of the train by getting the signals through the antenna. The GPS is connected to the satellite through the antenna and obtain the data in a format that is acceptable by the microcontroller. This is given to the microcontroller through the RS-232 which converts the incoming signal in a form of logic level data. The microcontroller used here is of 8051 architecture. Microcontroller is provided with a speed control[3-4] algorithm whose sole purpose is to provide the control the speed by informing the driver to increase or decrease the speed through a visual unit.

With the help of database and a calculation algorithm, the speed and position is calculated and a message is passed about the same on to the zigbee transmitter. At the control station we have a zigbee receiver which receives the incoming signal and is displayed on the display system through Microcontroller.

\section{OPERATING PRINCIPLe}

\subsection{On Train}

\subsubsection{Mode 1}

a. T Switch ON the Respective Track Switch whose information is needed.

b. Get the data from RBC which is in the format S-5-3-L-3-1-R-2-1-S-6-3

(Direction-Distance to be travelled-Speed to be maintained) Store the data

c. Get the GPS data, decode the data and store the Start_Latitude, Start_Longitude, Departure_Time data of the starting point and then Start the Train.

d. Initialize Position to 2, Speed to 3 .

e. Get the GPS data, decode the data and store the Previous_Latitude, Previous_Longitude, Previous_Time data.

f. Distance_to be_travelled $=($ Position +3$) * 10$ Speed_to_be_maintained $=($ Speed +3$) * 10$ 
g. Get the GPS data, decode the data and store the New_Latitude, New_Longitude, New_Time data

h. Calculate the Current_Distance i.e. using the formula given below

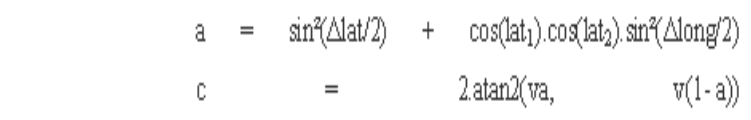

Haversine formula: $d=R . c$

Where $R$ is eath's radius (meen radius $=6,377 \mathrm{~km})$;

note that omgles need to be in radicus to pass to trig functions!

Current_Time $=($ Previous_Time - New_time $)$

Current_Speed $=($ Current_Distance / Current_time $)$

Difference_In_Speed $=($ Current_Speed $\sim$ Speed_to_be_maintained $)$

i. If Difference in Speed is not equal to zero Go to step j else Go to step $\mathrm{k}$

j. $\quad$ Display the Difference_In_Speed and Advice the Driver and Send the Information about Position and Difference_In_Speed to RBC in the Format : 02-23-00

(Position - Difference_In_Speed - Braking Zone)

k. If the Current_Distance is equal to Distance_to be_travelled Go to Step d else Go to Step g.

\subsubsection{Mode 2}

a. Receive the Track Signal which will be in the Format Z-1,2 ....3-R/Y/G .Store the Data

b. Get the data from RBC which is in the format S-5-3-L-3-1-R-2-1-S-6-3

(Direction-Distance to be travelled- Speed to be maintained) Store the data

c. Initialize Position to 2, Speed to 3, Current_Zone to 0, Braking_Zone to 0.

d. Store the Status of the First 4 Consecutive Zones in LED[ ]. Ex: LED [4] $=\{$ GGRG $\}$

e. Get the GPS data, decode the data and store the Previous_Latitude, Previous_Longitude, Previous_Time data.

f. Distance_to be_travelled $=($ Position +3$) * 10$

Speed_to_be_maintained $=($ Speed +3$) * 10$

g. Get the GPS data, decode the data and store the New_Latitude, New_Longitude, New_Time data

h. Display and Store the Status of the FOUR Consecutive Zones from Current_Zone in LED[ ].

Ex: LED $[4]=\{$ GGRG $\}$

i. If LED [i]=R then make LED $[\mathrm{i}-1]=\mathrm{Y}$

j. $\quad$ Ex: LED $[4]=\{$ GYRG $\}$

$\mathrm{k}$. If any of the LED variable status is $\mathrm{Y}$ or $\mathrm{R}$ store the position of the Zone in Braking_Zone.

1. If Current_Zone $=$ Braking_Zone then go step 0

m. Calculate the Current_Distance i.e using the formula given below

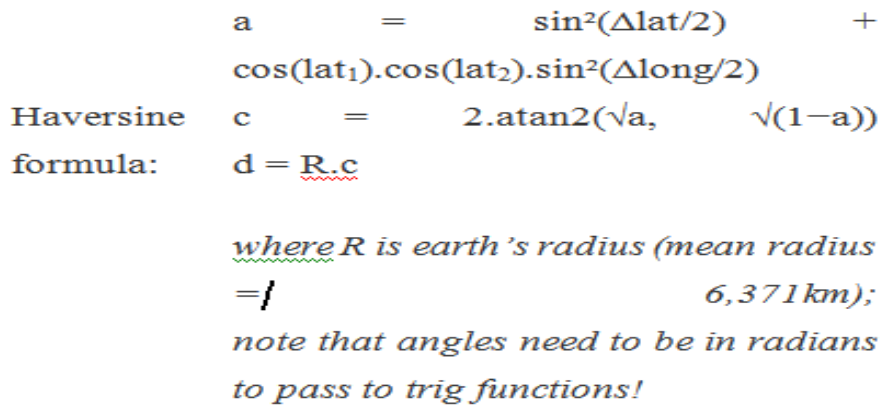

Current_Time $=($ Previous_Time - New_time $)$

Current_Speed $=($ Current_Distance / Current_time $)$

n. If the Current_Distance is equal to Distance_to be_travelled

o. Increment Current_Zone then Go to Step e else Go to Step g.

p. Apply brakes at the respective Zone and send the information about the BRAKES APPLIED and Stop. 
1.13 Mode 3

Switch ON the Respective Track Switch whose information is needed.

a. Get the data from RBC which is in the format S-5-3-L-3-1-R-2-1-S-6-3 (Direction-Distance to be travelled- Speed to be maintained)Store the data

b. Initialize Position to 2 .

c. Get the GPS data, decode the data and store the Previous_Latitude, Previous_Longitude, Previous_Time data

d. Distance_to be_travelled $=($ Position +3$) * 10$

e. Get the GPS data, decode the data and store the New_Latitude, New_Longitude, New_Time data

f. Calculate the Current_Distance i.e using the formula given below $\mathrm{a}=\sin ^{2}(\Delta \mathrm{lat} / 2)+\cos \left(\mathrm{lat}_{1}\right) \cdot \cos \left(\mathrm{lat}_{2}\right) \cdot \sin ^{2}(\Delta \mathrm{long} / 2)$

$\begin{array}{lll}\text { Haversine } & \mathrm{c} & = \\ \text { formula: } & \mathrm{d}=\mathrm{R} . \mathrm{c}\end{array}$

where $R$ is earth's radius (mean radius $=6,371 \mathrm{~km}$ ); note that angles need to be in radians to pass to trig functions!

Current_Time $=($ Previous_Time - New_time $)$

Current_Speed $=($ Current_Distance / Current_time $)$ g. Send position

\subsection{At RBC}

a. Initialize Gate to 0

b. Receive and decode the data

c. If the request is to send the Track information send the same from the database

d. Else if the data is in the Format 02-23-0 (Position - Difference_In_Speed - Braking Zone) Decode the Data to get the Position, Difference_In_Speed, Braking Zone

e. Display the Position of the Train.

f. If Position $=$ Gate_Position and Gate $=0$ send Close to Level Crossing and make Gate $=1$

g. If Position = Gate_Position and Gate $=1$ send Open to Level Crossing and make Gate $=0$

h. If Braking Zone is != 0 Display the Braking Postion

i. If Difference_In_Speed is != 0 Display the Difference_In_Speed else Go to Step b

\section{CONCLUSION}

It is used to avoid accidents in railways and saves the lives of a large number of passengers in the train. The GPS receives signals from a group of three satellites for getting the latitude and longitude of the object which is the train in our case. The data is precise and is always compatible with real time systems as the satellites are continuously monitoring. If we carefully set up the GPS system with précised parameters, the person in the control station will be able to track the position and speed of the train. This module gives the information related to the train and will alert the train driver whether to continue with the same speed or make any changes in the speed. Accordingly the control station will receive the information about the speed [5] and position of the train.

The module can have an additional feature of having the database that stores the information about the speed at various stages which can be used for investigation purposes in case of accidents. Changing the speed can be made automatic rather than manually. In case of emergency send the information about the position of train to Helping Authorities. Controlling of the speed can be made automatic. Information can be updated in communication media such as Internet. A lay man or a common man can access the information about the train through the devices like mobiles.

\section{Acknowledgements}

The authors thank the reviewers for their valuable comments and suggestions that helped us to make the paper in its present form 


\section{REFERENCES}

\section{Journal Papers:}

[1] Dai, Ling-Long; Wang, Zhaocheng ; Yang, Zhi-Xing, Time-Frequency Training OFDM with High Spectral Efficiency and Reliable Performance in High Speed Environments, IEEE Journal on Selected Areas in Communications, Volume: 30 , Issue: 4, 695 - 707

\section{Proceedings Papers:}

[2] BuBing, Wang, XiShi, Wu, XinYao, Wireless data transmission for high-speed train control, Vehicular Technology Conference, 2000. IEEE-VTS Fall VTC 2000. 52nd, Vol : 6, Pg : 2998 -3001

[3] Mei, T. X. ; Li, H., , Monitoring train speed using bogie mounted sensors - Accuracy and robustness, IET International Conference on Railway Condition Monitoring, 2008 , Pg:1 - 6

[4] Wei, ShangGuan ; Cai Bai-gen ; Gou Chen-xi ; Chen Jian-qiu ; Wang Jian, ,Research on key techniques of high-speed train control system simulation \& testing, International Conference on Mechatronics and Automation (ICMA) 2010, Page(s): 1695 - 1700

[5] Bing, $\mathrm{Bu}$; Xishi, Wang, The effect of speed on error rate in wireless train control Proceedings of the 3rd World Congress on Intelligent Control and Automation, 2000, vol.4, Pg : 2484 - 2487 